\title{
Clinical characteristics of coronavirus disease 2019 patients with diarrhea in Daegu
}

\author{
Min Kyu Kang, Kyeong Ok Kim, Min Cheol Kim, Joon Hyun Cho, Sung Bum Kim, Jung Gil Park, \\ Kook Hyun Kim, See Hyung Lee, Byung Ik Jang, and Tae Nyeun Kim
}

Department of Internal Medicine, Yeungnam University College of Medicine, Daegu, Korea
Background/Aims: Coronavirus disease 2019 (COVID-19) can reportedly cause gastrointestinal symptoms. Therefore, we investigated the clinical characteristics of COVID-19 patients with diarrhea.

Methods: We included 118 COVID-19 patients admitted to a single hospital from February 20 to March 31, 2020. Medical records with clinical characteristics, laboratory data, treatment course, and clinical outcomes were compared based on the presence or absence of diarrhea. Prognostic factors for disease severity and mortality in COVID-19 were also assessed.

Results: Among patients, 54 (45.8\%) had diarrhea, whereas seven (5.9\%) had only diarrhea. The median age of patients with diarrhea was 59 years (44 to 64), and 22 (40.7\%) were male. Systemic steroid use, intensive care unit admission, septic shock, and acute respiratory distress syndrome were less frequent in the diarrhea group than in the non-diarrhea group. No significant differences were observed in total hospital stay and mortality between groups. On multivariate analysis, age (odds ratio [OR], 1.06; 95\% confidence interval [CI], 1.01 to 1.12; $p=0.044$ ), diabetes (OR, 3.00; 95\% CI, 1.25 to 20.47; $p=0.042$ ), and dyspnea (OR, 41.19; 95\% CI, 6.60 to 823.16; $p<0.001)$ were independent risk factors for septic shock. On Cox regression analysis, diabetes (hazard ratio [HR], $4.82 ; 95 \% \mathrm{CI}, 0.89$ to $26.03 ; p=0.043$ ) and chronic obstructive pulmonary disease (HR, 16.58; 95\% CI, 3.10 to 88.70; $p=0.044$ ) were risk factors for mortality.

Conclusions: Diarrhea was present in $45.8 \%$ of patients and was a common symptom of COVID-19. Although patients with diarrhea showed less severe clinical features, diarrhea was not associated with disease severity or mortality.

Keywords: COVID-19; Diarrhea; Shock, septic; Mortality

\section{INTRODUCTION}

The coronavirus disease 2019 (COVID-19) outbreak initially occurred in Wuhan, Hubei province of China [1]. After the first outbreak, Daegu, the third largest city in South Korea, became the second outbreak hotspot in the world on February 20, 2020. Since then, COVID-19 has been declared a pandemic, thereby becoming a threat to global health and worsening socio-economic conditions [2-4].

COVID-19 is caused by the severe acute respiratory syndrome coronavirus 2 (SARS-CoV-2) [5]. This virus can cause serious respiratory disease, and its diagnosis and treatment have been mainly focused on respiratory symptoms such as fever, chills, cough, and sputum production. However, a recent Chinese multicenter study 
reported that the prevalence of extrapulmonary symptoms such as gastrointestinal symptoms was not insignificant [6], and some other studies have reported the prevalence of gastrointestinal symptoms to be approximately 50\% in COVID-19 patients [6,7]. The gastrointestinal symptoms associated with COVID-19 are diarrhea, anorexia, nausea, and vomiting. Among these, diarrhea is one of the most frequently reported symptoms [6]. Since the presence or absence of nausea or discomfort might be subjective, diarrhea would be a more reliable symptom for assessment. Therefore, we investigated the prevalence, baseline clinical characteristics, therapy, and clinical outcomes, including mortality, of COVID-19 patients in Daegu who were classified according to the presence or absence of diarrhea. Additionally, we evaluated the prognostic factors and whether diarrhea could be a predictor of severe disease or mortality for COVID-19.

\section{METHODS}

\section{Patients}

From February 20 to March 31, 2020, 118 confirmed COVID-19 patients ( $>19$ years) were hospitalized at our institution. All patients underwent chest radiography and laboratory tests, including complete blood cell count, liver, and kidney function tests, with careful patient interviews on the day of admission. The medical records, which included data on age, sex, body mass index, epidemiological history such as comorbidities, clinical characteristics such as diarrhea and respiratory symptoms on admission, laboratory parameters, treatment course, and clinical outcomes such as mortality were retrospectively reviewed. Clinical outcomes were followed up until April 15, 2020. This study was approved by the Institutional Review Board of Yeungnam University Medical Center, and the requirement of written informed consent by the patients was waived owing to the retrospective nature of the study (IRB No: 2020-04-030).

\section{Definitions}

In accordance with the World Health Organization (WHO) interim guidelines, real-time polymerase chain reaction (PCR) from nasopharyngeal and/or oropharyngeal swabs was used to confirm COVID-19 cases. Spu- tum and/or endobronchial aspirate were obtained from patients with severe respiratory disease. Duration from symptom onset to admission was defined as the interval between the potential earliest date of symptom (fever, chill, cough, sputum production, diarrhea, myalgia, and headache) and the date of hospital admission at our institution. After the COVID-19 outbreak in Daegu, even asymptomatic COVID-19 patients were tested early for COVID-19 using real-time PCR. Therefore, we considered the duration from symptom onset to admission instead of the duration from symptom onset to test day for COVID-19 in this study.

Diarrhea was defined as the presence of any of the following criteria: (1) change in bowel habit to loose stools with frequency of diarrhea $>3$ times/day and (2) diosmectite use for symptom control. Duration of diarrhea was defined as the interval from the potential earliest date of diarrhea or diosmectite use for symptom relief to the recorded date of no diarrhea in medical charts. After admission, we included the patients' diosmectite use in their medical records.

Fever was defined as a tympanic temperature of $37.5^{\circ} \mathrm{C}$ or higher. Systemic inflammatory response syndrome (SIRS) on admission was defined as the presence of at least two symptoms: (1) body temperature $<36^{\circ} \mathrm{C}$ or $>$ $38^{\circ} \mathrm{C}$, (2) heart rate $>90$ beats $/ \mathrm{min},(3)$ tachypnea $>20$ breaths/min, and (4) white blood cell count $<4,000$ or $>12,000$ cells $/ \mathrm{mm}^{3}$ [8]. Shock and acute respiratory distress syndrome (ARDS) was defined in accordance with the WHO interim guidelines [9]. Acute kidney injury was defined as an increase in the serum creatinine level of $>$ $0.3 \mathrm{mg} / \mathrm{dL}$ within 48 hours or 1.5 times the baseline level within 7 days; and decreased urine output of $<0.6 \mathrm{~mL} /$ $\mathrm{kg} / \mathrm{hr}$ for 6 hours [10]. Patients who needed high oxygen including the necessity of mechanical ventilation and high-flow oxygen therapy were admitted to the intensive care unit (ICU). Abnormal parenchymal finding was defined as a ground glass appearance with hazy opacity and consolidation; this was detected using chest radiography and analyzed by an experienced chest radiologist.

\section{Statistical analysis}

All continuous variables were expressed as a mean with standard deviation (mean $\pm \mathrm{SD}$ ) or median with range. The clinical characteristics and outcomes were compared using Student's $t$ test or Mann-Whitney $U$ test 
for continuous variables. The categorical values were compared using chi-square test or Fischer's exact test. Factors related to septic shock were identified with univariate and multivariate logistic regression analysis. Cox proportional hazard regression analysis was used to assess prognostic factors for mortality. $p$ values $<0.05$ were considered statistically significant. All statistical analyses were performed using SPSS version 23.0 (IBM Co., Armonk, NY, USA).

\section{RESULTS}

\section{Baseline and clinical characteristics of patients}

Among the 118 COVID-19 patients, diarrhea accompanied other symptoms in 54 patients (45.8\%), whereas diarrhea was the only symptom in seven patients $(5.9 \%$ of all the COVID-19 patients and $13.0 \%$ of COVID-19 patients with diarrhea) (Fig. 1). The baseline characteristics of the patients are shown in Table 1. The median age was 61.o years (range, 50.0 to 70.0), and 52 subjects (44.1\%) were male. Among COVID-19 patients, 84 (71.2\%) had at least one comorbidity, such as hypertension (34.7\%) and diabetes mellitus (DM) (16.1\%). On admission, there were 77 (65.3\%) patients with fever or chills, 84 (71.2\%) with cough or sputum production, 49 (41.5\%) with dyspnea, 47 (39.8\%) with myalgia, 43 (36.4\%) with headache,

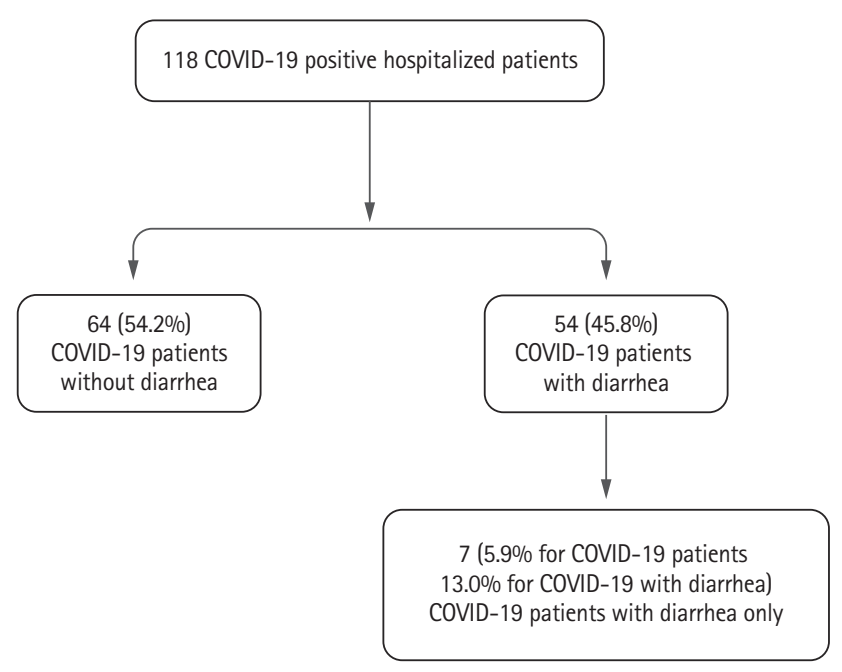

Figure 1. Flow chart of coronavirus disease 2019 (COVID-19) patients according to the presence of diarrhea. and seven (5.9\%) with only diarrhea. The mean duration from symptom onset to admission was $5.4 \pm 8.2$ days.

Patients were classified based on the presence or absence of diarrhea; there was no significant difference in baseline characteristics between the two groups. In the diarrhea group, the median age was 59.0 years (range, 44.0 to 64.0), and 22 (40.7) were male. The frequency of accompanying symptoms such as fever or chills and cough or sputum production were higher in the diarrhea group, but there were no significant differences (60.9\% vs. $70.4 \%$ with fever or chills, $p=0.380$; $68.8 \%$ vs. $74.1 \%$ with cough or sputum production). The mean duration from symptom onset to admission was $6.8 \pm$ 9.8 days in patients with diarrhea versus $4.1 \pm 6.3$ days in those without diarrhea $(p=0.086)$. The clinical manifestation showed differences in the respiratory rate (21.6 breaths/min in the non-diarrhea group vs. 20.5 breaths/ min in the diarrhea group, $p=0.021$ ) and initial oxygen saturation (93.5\% in the non-diarrhea group vs. $96.6 \%$ in the diarrhea group, $p=0.002$ ) (Table 1 ).

\section{Laboratory and radiologic results}

White blood cell count was lower in the diarrhea group than in the non-diarrhea group $\left(7,124 / \mathrm{mm}^{3}\right.$ vs. $5,843 /$ $\mathrm{mm}^{3}, \mathrm{p}=0.035$ ). Lymphocyte count was higher in the diarrhea group than in the non-diarrhea group (21.5\% vs. $27.0 \%, p=0.012)$. Patients in the diarrhea group had lower levels of blood urea nitrogen $(17.3 \mathrm{mg} / \mathrm{dL}$ vs. 13.1 $\mathrm{mg} / \mathrm{dL}, p=0.005)$ and serum potassium $(4.2 \mathrm{mEq} / \mathrm{L}$ vs. $4.0 \mathrm{mEq} / \mathrm{L}, p=0.033)$, and a higher estimated glomerular filtration rate (eGFR) $\left(86.7 \mathrm{~mL} / \mathrm{min} / 1.73 \mathrm{~m}^{2}\right.$ vs. $102.4 \mathrm{~mL} /$ $\left.\min / 1.73 \mathrm{~m}^{2}, p=0.004\right)$. There were no significant differences in the liver battery tests and C-reactive protein levels between the groups (Table 2).

Among all patients, 114 (96.6\%) had abnormal parenchymal findings, as detected by chest radiography. Interestingly, all patients with only diarrhea also showed abnormal parenchymal findings (data not shown). Similar proportions of abnormal chest radiography findings were noted in both groups (96.9\% vs. $96.3 \%, p=1.00$ ). Typical ground-glass opacity was the most common finding in 91 (85.1\%) patients. A total of 50 (80.6\%) and $47(90.4 \%)$ patients in the non-diarrhea and diarrhea groups, respectively, had ground-glass appearance $(p=$ o.234) (Table 2). 
Table 1. Baseline characteristics of the patients according to presence or absence of diarrhea

\begin{tabular}{|c|c|c|c|c|}
\hline Variable & $\begin{array}{c}\text { No diarrhea } \\
(\mathrm{n}=64,54.2 \%)\end{array}$ & $\begin{array}{c}\text { Diarrhea } \\
(\mathrm{n}=54,45.8 \%)\end{array}$ & $\begin{array}{c}\text { Total } \\
(\mathrm{n}=118,100.0 \%)\end{array}$ & $p$ value \\
\hline Age, yr & $62.5(50.0-72.0)$ & $59.0(44.0-64.0)$ & $61.0(50.0-70.0)$ & 0.079 \\
\hline Male sex & $30(46.9)$ & $22(40.7)$ & $52(44.1)$ & 0.629 \\
\hline $\mathrm{BMI}, \mathrm{kg} / \mathrm{m}^{2}$ & $24 \cdot 2 \pm 3 \cdot 3$ & $22.9 \pm 3.7$ & $23.6 \pm 3.5$ & 0.053 \\
\hline \multicolumn{5}{|l|}{ Comorbidities } \\
\hline Diabetes mellitus & $9(14.1)$ & $10(18.5)$ & $19(16.1)$ & 0.686 \\
\hline Hypertension & $26(40.6)$ & $15(27.8)$ & $41(34.7)$ & 0.205 \\
\hline Coronary artery disease & $5(7.8)$ & $4(7 \cdot 4)$ & $9(7.6)$ & 1.000 \\
\hline COPD & $6(9.4)$ & $2(3 \cdot 7)$ & $8(6.8)$ & 0.393 \\
\hline Chronic liver disease & $1(1.6)$ & $3(5.6)$ & $4(3 \cdot 4)$ & 0.494 \\
\hline Fatty liver & 0 & $2(3 \cdot 7)$ & $2(1.7)$ & 0.403 \\
\hline Chronic hepatitis C & o & $1(1.9)$ & $1(0.8)$ & 0.932 \\
\hline Malignancy & $3(4.8)$ & $5(9 \cdot 3)$ & $8(6.8)$ & 0.553 \\
\hline \multicolumn{5}{|l|}{ Initial symptoms on admission } \\
\hline Fever/chills & $39(60.9)$ & $38(70.4)$ & $77(65 \cdot 3)$ & 0.380 \\
\hline Cough/sputum & $44(68.8)$ & $40(74.1)$ & $84(71.2)$ & 0.666 \\
\hline Dyspnea & $30(46.9)$ & $19(35.2)$ & $49(41.5)$ & 0.273 \\
\hline Myalgia & $24(37 \cdot 5)$ & $23(42.6)$ & $47(39.8)$ & 0.708 \\
\hline headache & $21(32.8)$ & $22(40.7)$ & $43(36.4)$ & 0.484 \\
\hline Only diarrheal symptom & o & $7(13.0)$ & $7(5 \cdot 9)$ & 0.010 \\
\hline Duration from symptom onset to admission, day & $4.1 \pm 6.3$ & $6.8 \pm 9.8$ & $5.4 \pm 8.2$ & 0.086 \\
\hline Duration of diarrhea on admission, day & $0.0 \pm 0.0$ & $4.2 \pm 3 \cdot 0$ & & $<0.001$ \\
\hline \multicolumn{5}{|l|}{ Vital sign on admission } \\
\hline $\mathrm{SBP}, \mathrm{mmHg}$ & $134.0 \pm 20.5$ & $125 \cdot 7 \pm 17.8$ & $130.2 \pm 19.7$ & 0.023 \\
\hline Heart rate, beats/min & $85.1 \pm 18.7$ & $83.5 \pm 17.7$ & $84.4 \pm 18.2$ & 0.65 \\
\hline Body temperature, ${ }^{\circ} \mathrm{C}$ & $37.2 \pm 0.8$ & $37.4 \pm 0.7$ & $37.3 \pm 0.7$ & 0.176 \\
\hline $\mathrm{RR}$, breaths/min & $21.6 \pm 3.3$ & $20.5 \pm 1.7$ & $21.1 \pm 2.7$ & 0.021 \\
\hline Initial oxygen saturation, \% & $93 \cdot 5 \pm 7.5$ & $96.6 \pm 1.9$ & $94.9 \pm 5.9$ & 0.002 \\
\hline
\end{tabular}

Values are presented as median (range), number (\%), or mean \pm SD.

BMI, body mass index; COPD, chronic obstructive pulmonary disease; SBP, systolic blood pressure; RR, respiratory rate.

\section{Therapy and clinical outcomes}

There were no significant differences in the usage of lopinavir/ritonavir (93.8\% vs. $92.6 \%, p=1.000$ ) and hydroxychloroquine (89.1\% vs. $94.4 \%, p=0.475$ ) between the two groups. Systemic steroid (40.6\% vs. $20.4 \%, p$ $=0.030$ ) and extracorporeal membrane oxygenation ( $10.9 \%$ vs. $0.0 \%, p=0.034$ ) were needed more frequently in the non-diarrhea group. More patients in the non-diarrhea group needed ICU care (23.4\% vs. $1.9 \%, p=0.002)$. SIRS on admission was noted in $32(27.1 \%)$ patients. SIRS on admission was comparatively lesser in the diarrhea group, but there was no significant difference (34.4\% vs. $18.5 \%, p=0.085)$. During hospitalization, septic shock (20.3\% vs. $1.9 \%, p=0.005)$ and $\operatorname{ARDS}(15.6 \%$ vs. $0.0 \%$, $p=0.007$ ) developed less in the diarrhea group. However, no significant differences were observed in mortality (7.8\% vs. 1.9\%, $p=0.295$ ) and length of hospital stay (25.1 days vs. 26.8 days, $p=0.407$ ) between the groups (Table 3 ). 
Table 2. Laboratory and imaging data of the patients

\begin{tabular}{|c|c|c|c|c|}
\hline Variable & $\begin{array}{c}\text { No diarrhea } \\
(\mathrm{n}=64,54.2 \%)\end{array}$ & $\begin{array}{c}\text { Diarrhea } \\
(\mathrm{n}=54,45.8 \%)\end{array}$ & $\begin{array}{c}\text { Total } \\
(\mathrm{n}=118,100.0 \%)\end{array}$ & $p$ value \\
\hline \multicolumn{5}{|l|}{ Laboratory data on admission } \\
\hline White blood count, $/ \mathrm{mm}^{3}$ & $7,124 \cdot 4 \pm 2,883 \cdot 5$ & $5,843.2 \pm 3,648.4$ & $6,538.1 \pm 3,304.2$ & 0.035 \\
\hline Lymphocyte, \% & $21.5 \pm 11.5$ & $27.0 \pm 11.4$ & $24.0 \pm 11.7$ & 0.012 \\
\hline Hemoglobin, g/dL & $15.1 \pm 17.7$ & $13.1 \pm 1.5$ & $14.1 \pm 13.1$ & 0.377 \\
\hline Platelet, $10^{3} / \mu \mathrm{L}$ & $244.9 \pm 107.0$ & $233 \cdot 4 \pm 97 \cdot 4$ & $239.6 \pm 102.4$ & 0.543 \\
\hline C-reactive protein, $\mathrm{mg} / \mathrm{dL}$ & $6.6 \pm 9.0$ & $4.2 \pm 6.6$ & $5 \cdot 5 \pm 8.1$ & 0.106 \\
\hline Procalcitonin, ng/dL & $0.4 \pm 1.6$ & $0.1 \pm 0.1$ & $0.3 \pm 1.2$ & 0.103 \\
\hline AST, IU/L & $48.5 \pm 55.1$ & $40.2 \pm 34.2$ & $44.7 \pm 46.8$ & 0.322 \\
\hline ALT, IU/L & $35.2 \pm 47.2$ & $37.1 \pm 43 \cdot 4$ & $36.1 \pm 45 \cdot 3$ & 0.826 \\
\hline ALP, IU/L & $80.7 \pm 41.6$ & $79.8 \pm 38.5$ & $80.3 \pm 40.1$ & 0.904 \\
\hline GGT, IU/L & $32.1 \pm 35 \cdot 3$ & $39.2 \pm 39.0$ & $35 \cdot 6 \pm 37.1$ & 0.383 \\
\hline LDH, IU/L & $667.6 \pm 404.9$ & $567.5 \pm 187.4$ & $621.7 \pm 326.3$ & 0.093 \\
\hline Total bilirubin, mg/dL & $0.8 \pm 0.5$ & $0.9 \pm 0.4$ & $0.8 \pm 0.4$ & 0.845 \\
\hline Albumin, g/dL & $3.7 \pm 0.6$ & $3.8 \pm 0.5$ & $3.7 \pm 0.5$ & 0.060 \\
\hline PT-INR & $1.2 \pm 0.1$ & $1.1 \pm 0.1$ & $1.1 \pm 0.1$ & 0.055 \\
\hline Blood urea nitrogen, mg/dL & $17 \cdot 3 \pm 10.1$ & $13.1 \pm 5 \cdot 3$ & $15 \cdot 4 \pm 8.5$ & 0.005 \\
\hline Creatinine, mg/dL & $0.9 \pm 0.6$ & $0.8 \pm 0.2$ & $0.9 \pm 0.5$ & 0.062 \\
\hline $\mathrm{eGFR}, \mathrm{mL} / \mathrm{min} / 1.73 \mathrm{~m}^{2}$ & $86.7 \pm 28.7$ & $102.4 \pm 28.6$ & $93.7 \pm 29.6$ & 0.004 \\
\hline Sodium, mEq/L & $138.7 \pm 3.3$ & $138.7 \pm 2.8$ & $138.7 \pm 3.1$ & 0.741 \\
\hline Potassium, mEq/L & $4.2 \pm 0.5$ & $4.0 \pm 0.5$ & $4.1 \pm 0.5$ & 0.033 \\
\hline Abnormal finding on chest radiography & $62(96.9)$ & $52(96.3)$ & $114(96.6)$ & 1.000 \\
\hline Ground glass appearance & $50(80.6)$ & $47(90.4)$ & $91(85.1)$ & 0.234 \\
\hline
\end{tabular}

Values are presented as mean \pm SD or number (\%).

AST, aspartate aminotransferase; ALT, alanine aminotransferase; ALP, alkaline phosphatase; GGT, gamma-glutamyl transferase; LDH, lactate dehydrogenase; PT-INR, prothrombin time-international normalized ratio; eGFR, estimated glomerular filtration rate.

\section{Prognostic factors for septic shock and mortality in COVID-19 patients}

COVID-19 was less severe in patients with diarrhea than in those with non-diarrhea. In multivariate analysis, higher age (odds ratio [OR], 1.06; 95\% confidence interval [CI], 1.01 to 1.12; $p=0.044)$, DM (OR, 3.00; 95\% CI, 1.25 to $20.47 ; p=0.042$ ), and dyspnea (OR, 41.19; $95 \%$ CI, 6.60 to 823.16; $p<0.001$ ) were identified as independent risk factors for septic shock (Table 4). The presence of diarrhea was not a significant prognostic factor (OR, $0.24 ; 95 \% \mathrm{CI}, 0.05$ to $1.03 ; p=0.065$ ). In Cox proportional regression analysis, DM (hazard ratio [HR], 4.82; 95\% CI, 0.89 to $26.03 ; p=0.043)$ and chronic obstructive pulmonary disease (COPD) (HR, 16.58; 95\% CI, 3.10 to 88.70; $p=$
0.044) were risk factors for mortality (Table 5). The presence of diarrhea was not a prognostic factor for septic shock and mortality in patients with COVID-19.

\section{DISCUSSION}

To the best of our knowledge, this is the first study in the Korean population that has reported the extrapulmonary manifestations of COVID-19 in patients, after their classification on the basis of the presence or absence of diarrhea. COVID-19, caused by SARS-CoV-2, mainly causes respiratory symptoms including fever, chills, cough, and sputum production [6]. Therefore, pa- 
Table 3. Therapy and clinical outcomes of patients on admission

\begin{tabular}{|c|c|c|c|c|}
\hline Variable & $\begin{array}{c}\text { No diarrhea } \\
(\mathrm{n}=64,54.2 \%)\end{array}$ & $\begin{array}{c}\text { Diarrhea } \\
(\mathrm{n}=54,45.8 \%)\end{array}$ & $\begin{array}{c}\text { Total } \\
(\mathrm{n}=118,100 \%)\end{array}$ & $p$ value \\
\hline SIRS on admission & $22(34.4)$ & $10(18.5)$ & $32(27.1)$ & 0.085 \\
\hline \multicolumn{5}{|l|}{ Therapy } \\
\hline Oxygen therapy & $26(40.6)$ & $14(25 \cdot 9)$ & $40(33.9)$ & 0.137 \\
\hline Lopinavir/ritonavir & $60(93.8)$ & $50(92.6)$ & $110(93.2)$ & 1.000 \\
\hline Hydroxychloroquine & $57(89.1)$ & $5^{1}(94.4)$ & $108(91.5)$ & 0.475 \\
\hline Systemic steroid & $26(40.6)$ & $11(20.4)$ & $37(31.4)$ & 0.030 \\
\hline Diosmectite & 0 & $50(92.6)$ & $50(42.4)$ & 1.000 \\
\hline CRRT & $1(1.6)$ & 0 & $1(0.8)$ & 1.000 \\
\hline ECMO & $7(10.9)$ & 0 & $7(5.9)$ & 0.034 \\
\hline \multicolumn{5}{|l|}{ Clinical course } \\
\hline ICU care & $15(23.4)$ & $1(1.9)$ & $16(13.6)$ & 0.002 \\
\hline Septic shock & $13(20.3)$ & $1(1.9)$ & $14(11.9)$ & 0.005 \\
\hline ARDS & $10(15.6)$ & o & $10(8.5)$ & 0.007 \\
\hline AKI & $3(4 \cdot 7)$ & 0 & $3(2.5)$ & 0.306 \\
\hline \multicolumn{5}{|l|}{ Outcomes } \\
\hline Death & $5(7.8)$ & $1(1.9)$ & $6(5.1)$ & 0.295 \\
\hline Duration of hospital stay, day & $25.1 \pm 11.1$ & $26.8 \pm 10.6$ & $25.9 \pm 10.9$ & 0.407 \\
\hline
\end{tabular}

Values are presented as number (\%) or mean \pm SD.

SIRS, systemic inflammatory response syndrome; CRRT, continuous renal replacement therapy; ECMO, extracorporeal membrane oxygenation; ICU, intensive care unit; ARDS, acute respiratory distress syndrome; AKI, acute kidney injury.

Table 4. Independent risk factors for septic shock

\begin{tabular}{|c|c|c|c|}
\hline \multirow{2}{*}{ Variable } & \multirow{2}{*}{$\begin{array}{c}\text { Univariate } \\
\text { p value }^{\mathrm{a}}\end{array}$} & \multicolumn{2}{|c|}{ Multivariate analysis } \\
\hline & & $p$ value $^{a}$ & OR $(95 \% \mathrm{CI})$ \\
\hline Age, yr & $<0.001$ & 0.044 & $1.06(1.01-1.12)$ \\
\hline Male sex & 0.299 & & \\
\hline Overweight (BMI $\left.\geq 25 \mathrm{~kg} / \mathrm{m}^{2}\right)$ & 0.098 & & \\
\hline Diabetes mellitus & 0.002 & 0.042 & $3.00(1.25-20.47)$ \\
\hline Hypertension & 0.069 & & \\
\hline Chronic obstructive pulmonary disease & 0.034 & & \\
\hline History of cancer & 0.962 & & \\
\hline Fever/chills & 0.606 & & \\
\hline Cough & 0.983 & & \\
\hline Dyspnea & 0.002 & $<0.001$ & $41.19(6.60-823.16)$ \\
\hline Diarrhea & 0.016 & & \\
\hline Myalgia & 0.146 & & \\
\hline Headache & 0.015 & & \\
\hline
\end{tabular}

OR, odds ratio; CI, confidence interval; BMI, body mass index.

${ }^{\mathrm{a} C a l c u l a t e d}$ by logistic regression analysis. 
Table 5. Independent risk factors for death

\begin{tabular}{|c|c|c|c|}
\hline \multirow{2}{*}{ Variable } & \multirow{2}{*}{$\begin{array}{c}\text { Univariate } \\
\text { p value }^{\mathrm{a}}\end{array}$} & \multicolumn{2}{|c|}{ Multivariate analysis } \\
\hline & & $p$ value $^{\mathrm{a}}$ & $\operatorname{HR}(95 \% \mathrm{CI})$ \\
\hline Age, yr & 0.745 & & \\
\hline Male sex & 0.079 & & \\
\hline Overweight (BMI $\left.\geq 25 \mathrm{~kg} / \mathrm{m}^{2}\right)$ & 0.256 & & \\
\hline Diabetes mellitus & 0.031 & 0.043 & $4.82(0.89-26.03)$ \\
\hline Hypertension & 0.456 & & \\
\hline Chronic obstructive pulmonary disease & $<0.001$ & 0.010 & $16.58(3.10-88.70)$ \\
\hline History of cancer & 0.215 & & \\
\hline Fever/chills & 0.389 & & \\
\hline Cough & 0.781 & & \\
\hline Dyspnea & 0.084 & & \\
\hline Diarrhea & 0.174 & & \\
\hline Myalgia & 0.794 & & \\
\hline Headache & 0.482 & & \\
\hline
\end{tabular}

HR, hazard ratio; CI, confidence interval; BMI, body mass index.

${ }^{\mathrm{a} C a l c u l a t e d}$ by Cox proportional hazards regression test.

tient diagnosis and classification is based on the severity of respiratory symptoms and oxygen demand. However, according to a recent report, it is also possible for COVID-19 to manifest with only gastrointestinal symptoms like other viruses [6].

Several hypotheses have been proposed to understand why COVID-19 causes gastrointestinal symptoms including diarrhea. Molecular studies in the Chinese population have shown that there is an approximately $79 \%$ overlap in the sequences of SARS-CoV-2 and SARS$\mathrm{CoV}[11,12]$. Among these shared sequences, the angiotensin converting enzyme II (ACE2) receptor used for entry by the SARS-CoV was confirmed to be present in SARS-CoV-2 [11,12]. The ACE2 receptor is known to be highly expressed in lung alveolar type 2 cells, cholangiocytes, esophagus epithelial cells, and enterocytes in the ileum $[13,14]$. Entry of SARS-CoV-2 into the organs of the digestive system might be due to the increased permeability of the enterocytes, subsequently leading to diarrhea. Several studies have revealed that SARS-CoV-2 can be detected in stool samples in approximately 50\% of the patients [15-17]. In addition, owing to the systemic immune response to COVID-19, diarrhea may also be caused by the damage to the intestinal mucosa. However, it is difficult to confirm the sequential relationship in this study, and further large-scale studies are promptly needed.

Generally, viral enteritis is accompanied by nausea, vomiting, and anorexia as well as diarrhea; it runs a mild clinical course. In our study, gastrointestinal symptoms other than diarrhea were also noted in hospitalized COVID-19 patients. However, gastrointestinal symptoms other than diarrhea mainly occurred after the administration of drugs such as lopinavir/ritonavir and hydroxychloroquine on admission, and the possibility of side effects due to drugs could not be ruled out.

In our study, there were no significant differences except for those in blood urea nitrogen, eGFR, and serum potassium between the two groups. These laboratory findings are associated with dehydration. Therefore, we could assume that the diarrhea in COVID-19 patients was not severe. Furthermore, steroid use, ICU care, septic shock, and ARDS were also less common in the diarrhea group than in the non-diarrhea group. The overall clinical course of patients in the diarrhea group was likely to be milder than that in the non-diarrhea group, but the mortality was not significantly different between the two groups.

Another study in China showed opposite results for the comparison of clinical courses [6]. We could not ex- 
plain the exact cause of this discrepancy clearly, but it may be attributable to the severity and duration of diarrhea. All patients with diarrhea showed mild severity and a self-limited course. Alternatively, this could be associated with the limitation of our study design. Since this was a retrospective single center study, there could be several biases. Considering that the average duration from symptom onset to admission was 6.8 days, the patients could not remember their symptoms exactly. In patients who were transferred from other hospitals after their conditions worsened, we could not conclusively determine either the presence of diarrhea at the time of admission, or the treatment course during the stay at secondary-care hospitals.

In this study, old age, DM, and dyspnea were independent risk factors for septic shock in patients with COVID-19. Moreover, DM and COPD were independent risk factors for mortality in COVID-19 patients. However, diarrhea was not associated with disease severity, as indicated by the rates of septic shock and mortality in this study.

This study has several other limitations. First, because it is a retrospective study conducted at a single tertiary-care hospital, it is difficult to generalize our results to the general population. Moreover, there is the possibility of selection bias. Since our hospital is a tertiary university hospital, the COVID-19 patients would present with more severe symptoms than patients at less specialized centers. Since the occurrence of the COVID-19 outbreak on February 18, 2020, the disinfection team of Daegu classified all COVID-19 patients based on severity, and patients with respiratory symptoms and/or those with high oxygen demand were transferred to a tertiary-care hospital preferentially, as per the city's policy. Second, because both clinicians and patients paid more attention to respiratory symptoms initially, most patients had difficulty in remembering the onset of diarrhea and their accompanying symptoms. This may have introduced recall bias, making it difficult to estimate the amount of stool, the exact duration from the onset of diarrhea to admission, as well as the presence of other gastrointestinal symptoms including nausea and vomiting. Third, because of the very small number of events, it is difficult to ascertain the association of the presence of diarrhea with disease severity and mortality. Despite these limitations, we thought that it could be clinically significant in that, we tried to evaluate the frequency and clinical significance of diarrhea in patients with COVID-19 at early phase of outbreak.

In conclusion, diarrhea could also be one of the main symptoms in COVID-19 patients. Although patients without diarrhea had a more severe clinical course than those with diarrhea, the presence of diarrhea may not be associated with disease severity and mortality. Multicenter studies with large numbers of patients are warranted to elucidate the clinical impact of diarrhea as a prognostic factor for COVID-19 patients.

\section{KEY MESSAGE}

1. Diarrhea presented along with primary symptoms in approximately 50\% of all hospitalized coronavirus disease 2019 (COVID-19) patients.

2. In patients with COVID-19, old age, diabetes mellitus (DM), and dyspnea are independent risk factors for septic shock and DM and chronic obstructive pulmonary disease are independent risk factors for mortality.

3. Presence of diarrhea in COVID-19 patients may not be associated with septic shock and mortality.

\section{Conflict of interest}

No potential conflict of interest relevant to this article was reported.

\section{Acknowledgments}

This study was supported by a research grant from the Daegu Medical Association COVID-19 Scientific Committee.

\section{REFERENCES}

1. Gu J, Han B, Wang J. COVID-19: gastrointestinal manifestations and potential fecal-oral transmission. Gastroenterology 2020;158:1518-1519.

2. Khot WY, Nadkar MY. The 2019 novel coronavirus outbreak: a global threat. J Assoc Physicians India 2020;68:6771.

3. Legido-Quigley H, Asgari N, Teo YY, et al. Are high-performing health systems resilient against the COVID-19 
epidemic? Lancet 2020;395:848-850.

4. Wang P, Anderson N, Pan Y, et al. The SARS-CoV-2 outbreak: diagnosis, infection prevention, and public perception. Clin Chem 2020;66:644-651.

5. Phelan AL, Katz R, Gostin LO. The novel coronavirus originating in Wuhan, China: challenges for global health governance. JAMA 2020;323:709-710.

6. Pan L, Mu M, Yang P, et al. Clinical characteristics of COVID-19 patients with digestive symptoms in Hubei, China: a descriptive, cross-sectional, multicenter study. Am J Gastroenterol 2020;115:766-773.

7. Gao QY, Chen YX, Fang JY. 2019 Novel coronavirus infection and gastrointestinal tract. J Dig Dis 2020;21:125-126.

8. Bone RC, Balk RA, Cerra FB, et al. Definitions for sepsis and organ failure and guidelines for the use of innovative therapies in sepsis. The ACCP/SCCM Consensus Conference Committee. American College of Chest Physicians/ Society of Critical Care Medicine. Chest 1992;101:16441655 .

9. World Health Organization. Clinical management of severe acute respiratory infection (SARI) when COVID-19 disease is suspected: interim guidance [Internet]. Geneva (CH): WHO, c2020 [cited 2020 Sep 10]. Available from: https://www.who.int/docs/default-source/coronaviruse/ clinical-management-of-novel-cov.pdf?sfvrsn=bc7da517_10\&downl oad=true.

10. Stevens PE, Levin A; Kidney Disease: Improving Global Outcomes Chronic Kidney Disease Guideline Develop- ment Work Group Members. Evaluation and management of chronic kidney disease: synopsis of the kidney disease: improving global outcomes 2012 clinical practice guideline. Ann Intern Med 2013;158:825-830.

11. Lu R, Zhao X, Li J, et al. Genomic characterisation and epidemiology of 2019 novel coronavirus: implications for virus origins and receptor binding. Lancet 2020;395:565574 .

12. Zhou P, Yang XL, Wang XG, et al. A pneumonia outbreak associated with a new coronavirus of probable bat origin. Nature 2020;579:270-273.

13. Zhang C, Shi L, Wang FS. Liver injury in COVID-19: management and challenges. Lancet Gastroenterol Hepatol 2020;5:428-430.

14. Zhang H, Kang Z, Gong $\mathrm{H}$, et al. The digestive system is a potential route of 2019-nCov infection: a bioinformatics analysis based on single-cell transcriptomes. BioRxiv. https://doi.org/10.1101/2020.01.30.927806.

15. Li M, Wang B, Zhang M, et al. Symbiotic gut microbes modulate human metabolic phenotypes. Proc Natl Acad Sci U S A 2008;105:2117-2122.

16. Tang A, Tong ZD, Wang HL, et al. Detection of novel coronavirus by RT-PCR in stool specimen from asymptomatic child, China. Emerg Infect Dis 2020;26:1337-1339.

17. Xie C, Jiang L, Huang G, et al. Comparison of different samples for 2019 novel coronavirus detection by nucleic acid amplification tests. Int J Infect Dis 2020;93:264-267. 